\title{
Transfistula guidewire for easier repair of esophageal
} atresia

\begin{abstract}
The aim of this report is to describe a helpful new technique for use in the management of congenital esophageal atresia with tracheoesophageal fistula. Before reconstruction of the esophagus, a bronchoscopy is performed to visualize the tracheal opening of the tracheoesophageal fistula. If the fistula can be identified, a guidewire is passed via the bronchoscope through the fistula, into the distal esophagus, and then into the stomach. The tracheoesophageal guidewire can then serve as a landmark to facilitate the esophageal reconstruction.
\end{abstract}

Keywords: esophageal atresia (EA), tracheoesophageal fistula (TEF), guidewire, newborn, bronchoscopy

\author{
Volume 4 Issue 2 - 2017
}

\author{
Anderberg M, Börjesson A, Stenström P, \\ Backman T,Arnbjörnsson E \\ Department of Pediatric Surgery, Lund University, Skåne \\ University Hospital, Sweden
}

Correspondence: Arnbjörnsson E, Department of Pediatric Surgery, Lund University, Skåne University Hospital, , Lund, Sweden, Email einar.arnbjornsson@telia.com

Received: January 17, 2017 | Published: April 03, 2017

\section{Introduction}

Dissection of the tracheoesophageal fistula (TEF) during reconstruction of a congenital esophageal atresia (EA) malformation by open thoracotomy or thoracoscopy is challenging. The reconstruction procedure includes clarifying the anatomy before dividing the TEF and anastomosing the proximal and distal ends of the esophagus. Identifying the TEF can be difficult. The aim of this report is to describe a technique for an approach that includes bronchoscopic placement of a guidewire through the TEF and into the distal portion of the esophagus to facilitate early identification and dissection of the TEF.

\section{Details of the proposed technique}

Bronchoscopy is usually integrated as an initial part of reconstructive surgery for EA. In cases where the TEF can be seen by bronchoscopy, it is may be useful to pass a guidewire through the fistula and into the distal part of the esophagus and the proximal stomach (Figure 1). The guidewire position can be verified by X-ray. The guidewire can be left in the distal part of the esophagus during thoracotomy or thoracoscopy. The presence of the guidewire can assist the operating surgeon with direct localization of the fistula without any unnecessary dissection in the thorax. The operation may be easier when the distal end of the esophagus and the TEF are marked by the presence of the guidewire. This technique should also be of use in laparoscopic and robot-or computer-assisted surgeries. We have routinely used a transfistula guidewire when repairing anorectal malformation (ARM) with recto urinary fistula. During ARM repair, the guidewire is passed through the divergent stoma down to the rectum, through the fistula to the urethra, and out through the urethral meatus. ${ }^{1}$ In a similar manner, during repair of EA with TEF, passing a guidewire from the trachea through the fistula and into the esophagus can facilitate the identification of the TEF. We believe this proposal merits discussion and, in the future, clinical trials should be planned. This is possible because bronchoscopy is now routinely used as the first intervention when treating newborns with EA at our center. However, there are potential drawbacks. Bronchoscopy takes time that may not be justified in cases where the TEF can be quickly and easily identified during surgery. Placing the guidewire through the TEF also takes time, and, as with all operative interventions, it is hampered by the risk of injury to the organs involved. Furthermore, the presence of the thin guidewire does not necessarily facilitate the localization of the fistula, and the guidewire may be also troublesome for the anesthesiologist who is managing the airway.

To test the idea here presented in a randomized study, we would need a larger population than the 6 to 8patients with EA that we have treated yearly at our center during the past two years. Since we have not yet routinely used this technique, we cannot yet claim any statistically significant gain associated with it. Our idea is hereby published to encourage input and opinions from others.

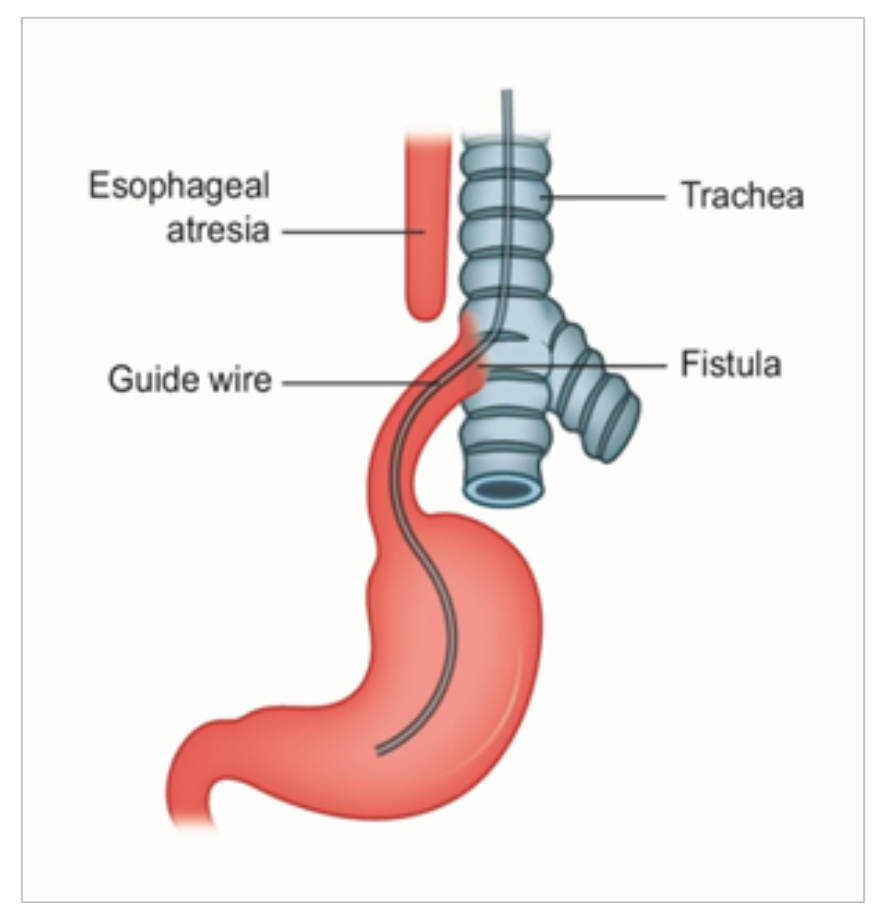

Figure I A schematic drawing of the guide wire passed from the trachea through the fistula to the esophagus. 


\section{Acknowledgments}

BioMed Proofreading LLC for the edited manuscript, and to Elsevier Web Shop for the illustration.

\section{Conflict of interest}

The author declares no conflict of interest.

\section{References}

1. Pernilla Stenström, Magnus Anderberg, Christina Clementson Kockum, et al. Endoscopically placed recto urethral guidewire facilitates the reconstruction of anus in children with anorectal malformations: a case report. Eur J Pediatr Surg Rep. 2013;1(1):46-47. 